\title{
Combination of Treosulfan, Fludarabine and Cytarabine as Conditioning in Patients With Acute Myeloid Leukemia, Myelodysplastic Syndrome and Myeloproliferative Neoplasms
}

\section{Samantha O'Hagan Henderson}

Klinikum Oldenburg AoR

Jochen J Frietsch ( $\sim$ jochen.frietsch@med.uni-jena.de )

Universitatsklinikum Jena https://orcid.org/0000-0002-1476-8059

Inken Hilgendorf

Jena University Hospital: Universitatsklinikum Jena

Andreas Hochhaus

Jena University Hospital: Universitatsklinikum Jena

Claus-Henning Köhne

Klinikum Oldenburg AoR

Jochen Casper

Klinikum Oldenburg AoR

\section{Research Article}

Keywords: AML, MDS, MPN, allogeneic stem cell transplantation, toxicity-reduced conditioning

Posted Date: July 26th, 2021

DOl: https://doi.org/10.21203/rs.3.rs-731909/v1

License: (c) (i) This work is licensed under a Creative Commons Attribution 4.0 International License.

Read Full License 


\section{Abstract}

Treosulfan and fludarabine (Treo/Flu) were successfully introduced into toxicity-reduced conditioning for SCT. However, the risk of post-SCT relapse remains a matter of concern. We report the results of a novel individual treatment approach with Treo/Flu and cytarabine (Treo/Flu/AraC) conditioning prior to allogeneic SCT in patients with acute myeloid leukemia (AML), myelodysplastic syndrome (MDS), or myeloproliferative neoplasms (MPN).

Seventy-seven patients (median age 54 years) at high risk of disease relapse due to unfavourable cytogenetics or failure to achieve complete remission prior to SCT were included. Median follow-up was 3.2 years. One-, two- and three-year RFS rates were $47.5 \%, 40.7 \%$, and $37.3 \%$ and OS rates were $59.3 \%$, $49.3 \%$, and $45.4 \%$, respectively. Cumulative incidence of NRM was $10 \%$ at 100 days, $18.8 \%$ at one year and $20.1 \%$ at two years. The cumulative incidence of relapse increased from $34 \%$ at one year to $41 \%$ after three years. The cumulative incidences of engraftment, chimerism, Graft-versus-host-disease (GvHD) and toxicities were acceptable and comparable with similar patients conditioned with Treo/Flu or FLAMSARIC.

In conclusion, Treo/Flu/AraC provides tolerable, feasible and effective conditioning for patients with AML, MDS or MPN, even in advanced disease states. The incidence of NRM and relapse is acceptable in this heavily pre-treated population with high-risk disease. Future research will aim to confirm these initial findings and include a larger number of participants in a prospective trial.

\section{Purpose}

Patients with active disease before allogeneic stem cell transplantation (SCT) or high risk of relapse are faced with a poor prognosis. The intensity of conditioning can heavily influence patient outcome. Myeloablative protocols reduce the risk of disease relapse compared to reduced intensity conditioning (RIC), but high non-relapse mortality (NRM) rates are a concern [1]. RIC has made allogeneic SCT accessible to those previously deemed unfit.

The first study to demonstrate the feasibility of conditioning with treosulfan and fludarabine (Treo/Flu) was published in 2004 [2]. Treosulfan is an alkylating agent with acceptable extra-medullary toxicity at doses up to $46 \mathrm{~g} / \mathrm{m}^{2}$ [3]. It has pronounced in vitro committed and non-committed hematopoietic stem cell toxicity [4] and in vitro anti-leukemic cell activity [5]. Fludarabine is a purine analogue that inhibits DNA and RNA synthesis [6]. Fludarabine has replaced cyclophosphamide in many conditioning regimens due to its improved toxicity profile [7].

Treo/Flu conditioning has been introduced into the management of patients with AML, MDS, and MPN [812]. European Society for Blood and Marrow Transplantation (EBMT) registry data have been used to compare Treo/Flu with other conditioning regimens such as FLAMSA-RIC (fludarabine, amsacrine and cytarabine) and busulfan-based regimens in patients with AML or MDS. Lower rates of acute graftversus-host disease (aGvHD) were seen in those treated with Treo/Flu [13, 14]. However, when Treo/Flu 
was compared to FLAMSA-TBI, the latter had a decreased risk of relapse and better leukemia-free survival [14]. A recent randomised phase III clinical trial compared the outcomes of patients with AML or MDS undergoing allogeneic SCT with either Treo/Flu or Bu/Flu conditioning. This study demonstrated the noninferiority of Treo/Flu compared to Bu/Flu [15]. Two-year overall survival (OS), transplant-related mortality and NRM were all significantly better after Treo/Flu conditioning. Event-free survival was better in the Treo/Flu group, but did not reach significance.

Cytarabine, a pyrimidine analogue, is a chemotherapeutic backbone in the treatment of haematologic malignancies. It is one of the most effective drugs for the treatment of AML, even after disease relapse $[16,17]$. When fludarabine is infused prior to the administration of cytarabine, the intracellular accumulation of the biologically active form of cytarabine is potentiated [18].

By combining cytarabine, an effective drug in the treatment of myeloid malignancies, with a well-tolerated and effective reduced toxicity conditioning regimen (Treo/Flu), the aim was to achieve better outcomes for patients transplanted with active disease and those with a high risk of relapse. The Treo/Flu/AraC regimen has potentially very strong anti-leukemic, immunosuppressive and cytotoxic effects. This retrospective study evaluates the outcomes of patients with AML, MDS, or MPN following conditioning with Treo/Flu/AraC in comparison to Treo/Flu prior to allogeneic SCT to evaluate the additive effect of AraC.

\section{Patients And Methods}

Eighty transplantations using this regimen were performed between July 2009 and August 2018 at the University Hospitals of Jena and Oldenburg in Germany. 77 patients ( $32 \%$ female) with AML, MDS, and MPN between 18 and 69 years of age (median 54 years) were conditioned with Treo/Flu/AraC (see Table 1 for patient and donor characteristics). Three patients received two transplantations with Treo/Flu/AraC conditioning. Patients were followed up until June 2019. The decision to give Treo/Flu/AraC was based on comorbidities, previous response to therapy and disease status. Patients gave written informed consent.

Fifty-two patients received treosulfan (Medac, Wedel, Germany) $14 \mathrm{~g} / \mathrm{m}^{2}$ intravenously over two hours from day -6 to -4 . Twenty-eight patients received treosulfan on day -4 to -2 . Fludarabine (Schering, Berlin, Germany) $30 \mathrm{mg} / \mathrm{m}^{2}$ was given intravenously over 30 minutes from day -6 to -2 . Cytarabine (various manufacturers) was administered at a dose of $2000 \mathrm{mg} / \mathrm{m}^{2}$ once daily over three hours on day -6 to -5 . Rabbit antithymocyte globulin (Neovii, Graefelfing, Germany) was given only for patients receiving grafts from unrelated donors as previously described [2].

\section{Supportive care}

Supportive care was given based on local guidelines. Prophylaxis against GvHD, consisting of ciclosporin A (CsA) and methotrexate, has been described elsewhere [19]. Whole-blood steady-state trough 
concentrations of CsA were maintained between $180 \mathrm{ng} / \mathrm{ml}$ and $230 \mathrm{ng} / \mathrm{ml}$. Based on its positive effect on patients' survival, ursodesoxycholic acid was administered daily to patients with rising serum bilirubin levels and in patients suspected of developing GvHD [20].

\section{Chimerism}

Chimerism was evaluated using PCR to amplify previously identified microsatellites in DNA extracts of peripheral blood mononuclear cells. Patients were classified as a complete chimera if the proportion of donor cells exceeded $98 \%$. Chimerism analysis was performed on day $+28,+100$ and +180 .

\section{Definition of primary and secondary outcomes and statistical analysis}

The primary endpoint was relapse-free survival (RFS) and this was calculated for each transplantation. The definition of RFS and analysis has been described previously [8].

Secondary endpoints were OS, NRM, cumulative incidence of relapse, engraftment and graft failure, chimerism, acute or chronic GvHD (aGvHD, cGvHD) and toxicities/adverse events as defined by the common terminology criteria for adverse events (CTCAE) version 5.0. Toxicities and adverse events were recorded during conditioning until day +28 .

OS was calculated from day 0 to death due to any cause and was analysed using the Kaplan-Meier method. Patients alive at their last follow-up were censored. NRM, relapse, engraftment, chimerism and aGvHD or cGvHD were estimated using cumulative incidence analysis considering competing risks. NRM was defined as death without previous relapse of disease. Relapse was a competing event. NRM was a competing event with regards to relapse. Engraftment of neutrophils and platelets were defined as described previously [9]. Primary graft failure [21], poor graft function [22] and secondary graft failure [23] have also been described elsewhere. Acute and chronic GvHD was classified according to the criteria of Harris et al. [24] and Filipovich et al. [25], respectively.

For exploratory purposes, outcome data (RFS, OS and NRM) were stratified by type of donor, remission status at transplantation (CR vs. all other statuses) and age ( $<50$ years vs. $\geq 50$ years). In the statistical analysis comparing groups, log-rank tests were used in Kaplan-Meier analyses and Gray's test was applied to cumulative incidence curves. A subgroup analysis of AML patients was also performed.

Median follow-up time was calculated using the method described by Schemper and Smith [26].

Statistical analysis using the Kaplan-Meier method was performed using SPSS (IBM Corp. 2017. Version 25.0. Armonk, NY, USA). Cumulative incidence curves with competing risk analysis and $95 \%$ confidence intervals were performed using $\mathrm{R}$ version 3.5.3 provided by the R Foundation. The method has been described elsewhere [27]. Statistical significance was defined at 0.05 . 


\section{Ethics and data protection}

The ethics committees of the Universities of Oldenburg and Jena approved the study in its current form (reference number Oldenburg: 2018-106, Jena: 2019-1316-BO). Analysis was performed on anonymised data and in accordance with the Declaration of Helsinki.

\section{Results}

At time of allogeneic SCT, the majority of patients $(55 \%, 44 / 80)$ were not in remission. The median percentage of bone marrow blasts at start of conditioning was $22.6 \%(\mathrm{Cl}(15 \%-30 \%))$ in these patients and 12 of those patients harboured the following mutations: FLT3-ITD (3), FLT3-ITD and NPM1 (2), FLT3ITD, FLT3-TKD and MLL (1), FLT3-TKD and MLL (1), IDH1, U2AF1 and MPL (1), MLL (1) and NPM1 (1). In contrast, 16 of the patients in CR harboured the following aberrations: FLT3-ITD (5), FLT3-ITD and NPM1 (6), FLT-TKD (1), NRAS and U2AF1 (1), RUNX1 (1), RUNX-RUNX1T1 and FIP1L1-PDGFRA (1), RUNXRUNX1T1 and FLT3-ITD (1). Median follow-up time was 3.2 years (range 13 days -9.8 years) after SCT.

\section{Relapse-free survival (RFS)}

One-, two- and three-year RFS was 47.5\%, 40.7\% and 37.3\% (see Figure 1). For matched-unrelated donor (MUD) transplantations, these figures were $46.9 \%, 38.0 \%$ and $35.4 \%$ and for matched-related donor (MRD) transplantations, 52.4\%, 47.1\% and 39.3\%, respectively. For mismatched unrelated donor (MMUD) transplantations, RFS was $40.0 \%$ at one year and remained at this level throughout follow-up. There was no significant difference between the groups $(p=0.76)$.

Patients transplanted in CR had a one-, two and three-year RFS of $48.6 \%, 37.5 \%$ and $34.1 \%$. The figures for those transplanted in non-CR were $46.5 \%, 41.5 \%$ and $38.0 \%(p=0.907)$.

Patients receiving a transplant at or over the age of 50 had a higher one-, two- and three-year RFS $(49.1 \%$, $43.1 \%$ and $40.2 \%)$ compared with patients younger than 50 years old $(44.4 \%, 37.0 \%$ and $33.3 \% ; p$ $=0.969)$ (see supplemental table S1).

One-, two- and three-year RFS rates for the AML subgroup analysis were $43.3 \%, 38.1 \%$ and $33.5 \%$.

\section{Overall survival (OS)}

One, two- and three-year OS was $59.3 \%, 49.3 \%$ and $45.4 \%$ (see Figure 1). For MRD transplantations, these figures were $70.0 \%, 48.1 \%$ and $40.1 \%$ and for MUD transplantations, these figures were $56.8 \%, 49.9 \%$ and $46.7 \%(p=0.95)$. The one-year OS for those receiving a MMUD was $48.0 \%$.

For patients transplanted in CR, the one-, two- and three-year OS was $65.6 \%, 53.3 \%$ and $45.1 \%$ and for those transplanted not in CR $54.3 \%, 46.2 \%$ and $46.21 \%(p=0.996)$, respectively (see supplemental table 
S2). The one-, two- and three-year OS for patients aged under 50 was $58.1 \%, 54.2 \%$ and $44.4 \%$ and for patients aged 50 and over $60.3 \%, 47.4 \%$ and $44.5 \%(p=0.785)$, respectively.

The one-, two- and three-year OS of the patients with AML was $56.5 \%, 48.9 \%$ and $43.5 \%$.

\section{Non-relapse mortality and cumulative incidence of relapse}

Eighteen patients were neutropenic before the start of conditioning and eight received conditioning despite radiological evidence of fungal pneumonia. Eighteen patients died without disease relapse. The causes of death of those patients who died before day +28 were: sinusoidal obstruction syndrome (1), sepsis (2) or pneumonia (1). The causes of death for the remaining fourteen patients were: sepsis/infection (7), acute respiratory distress syndrome (2), intracerebral bleed (1), post-transplant lymphoproliferative disorder (1), grade IV liver GvHD (1) or unknown (2).

Cumulative incidences of NRM were $10.0 \%(95 \% \mathrm{Cl}(5 \%, 18 \%))$ at 100 days, $18.8 \%(95 \% \mathrm{Cl}(11 \%, 28 \%))$ at 1 year and $20.1 \%(95 \% \mathrm{Cl}(12 \%, 30 \%))$ at 2 years (see Figure 2$)$. The one-, two- and three-year cumulative incidences of relapse were $34.0 \%(95 \% \mathrm{Cl}(24 \%, 44 \%)), 40.2 \%(95 \% \mathrm{Cl}(28 \%, 53 \%))$ and $41.0 \%(95 \% \mathrm{Cl}$ $(30 \%, 52 \%)$ ), respectively (see Figure 3$)$. The subgroup analyses for NRM and the cumulative incidence of relapse are depicted in table 2 and 3.

\section{Engraftment, graft failure and chimerism}

CTCAE grade IV neutropenia, leukocytopenia and thrombocytopenia occurred in all patients. Three patients died in the pre-engraftment phase. One patient achieved neutrophil engraftment, but died before platelet engraftment was achieved. The rest achieved engraftment of neutrophils and platelets.

The day 28 cumulative incidence of engraftment for neutrophils was $85.0 \%(95 \% \mathrm{Cl}(75 \%, 91 \%))$. By day +37 all patients had achieved successful neutrophil engraftment. The day 28 cumulative incidence of platelet engraftment was $82.5 \%(95 \% \mathrm{Cl}(72 \%, 89 \%))$. By day +100 , this had increased to $85.0 \%(95 \% \mathrm{Cl}$ $(75 \%, 91 \%))$.

The median time to neutrophil engraftment was 20 days (range 9-37 days), to platelet engraftment 20 days (range 11-174 days). No primary or secondary failure of engraftment was documented. There were nine cases of poor graft function with regards to neutrophil engraftment and twelve cases with regard to platelet engraftment.

The cumulative incidence of complete donor-type chimerism was $84.0 \%$ (95\% $\mathrm{Cl}(74 \%, 90 \%), 66$ subjects) on day +28 . By day $+100,80.0 \%$ of patients were found to have complete donor-type chimerism ( 55 subjects). By day +180 this figure fell to $68.0 \%$ (41 subjects). The course of chimerism mirrored the incidence of relapse. 


\section{Acute and chronic graft versus host disease}

Day 100 cumulative incidences of grade I-IV, II-IV and III-IV acute GVHD were $38.0 \%(95 \% \mathrm{Cl}(27 \%, 48 \%)$ ), $22.0 \%(95 \% \mathrm{Cl}(13 \%, 33 \%))$ and $6.0 \%(95 \% \mathrm{Cl}(2 \%, 14 \%))$. Two patients developed grade IV aGvHD of the liver. The cumulative incidence of mild to severe cGVHD at 2 years was $15.0 \%(95 \% \mathrm{Cl}(8 \%, 24 \%))$. There were three cases of severe cGvHD.

\section{Toxicities and adverse events}

Every patient experienced the expected chemotherapy related myelosuppression and required the transfusion of blood products following or prior to transplantation as a direct consequence of the conditioning chemotherapy. In some cases, myelosuppression was also caused by the underlying malignant condition or previous bridging or salvage therapy given prior to the start of conditioning. A detailed breakdown of recorded toxicities is depicted in table 4.

The diagnosis of sinusoidal obstruction syndrome (SOS) (grade 3) was suspected in two patients who suffered from progressive AML or from CML in transition to accelerated phase. A third patient suffering from progressive AML and renal insufficiency, who has received stem cells from a MMUD, likely died due to SOS (grade 5). The patients received SCT at the age of 44,55 , and 52 years from a matched-related donor, except for the younger AML patient, who had a MMUD. The HCT-Cl was 3, 0 and, 0 , respectively [28]. All three patients received the same fludarabine containing conditioning therapy as well as the same GvHD prophylaxis and none of them had received gemtuzumab ozogamicin, which is known to increase the risk of SOS [29].

Further adverse events included haemorrhagic colitis (1), neutropenic colitis (1), post-transplant lymphoproliferative disease (1), pericardial effusion with hemodynamic relevance (2), non-ST-segment elevation myocardial infarction (NSTEMI) (1), pulmonary embolism (1) and cardiac decompensation (2). Two patients developed a secondary malignancy. The first developed gallbladder cancer three years following a MMUD transplantation for AML. The second developed a ductal carcinoma in situ just over two and a half years following a MUD transplantation for CML.

\section{Conclusion}

This retrospective dual centre study evaluated the outcomes of patients with AML, MDS or MPN and high risk of relapse following conditioning with Treo/Flu/AraC prior to allogeneic SCT. The addition of cytarabine combined with the higher dose of Treosulfan $\left(14 \mathrm{~g} / \mathrm{m}^{2} \mathrm{vs} .10 \mathrm{~g} / \mathrm{m}^{2}\right.$ as used in [15]) aimed to enhance and optimise the antileukemic activity of this regimen.

To date, the majority of prospective Treo/Flu studies have selected AML patients in CR or MDS/CML patients with a low relapse risk $[15,19,30,31]$. This complicates cross-trial comparisons with the patients reported here. The patients in the current study either suffered from active disease or were in CR with high 
risk of relapse due to: (1) cytogenetic factors, (2) SAML, (3) post-salvage chemotherapy or (4) high-risk MPN (see Table 1). Many existing studies do not specify if salvage chemotherapy was required to achieve CR, instead relying on the statement, 'indication for allogeneic SCT according to institutional policy'. This description potentially hides a wide spectrum of differently responsive leukemic blasts and further hinders cross-trial comparisons.

In the current study, $43 \%$ of patients were not in CR at the time of transplantation, with a median blast count of $22.6 \%$. Secondary AML was diagnosed in $32 \%$ of patients. In the univariate analysis performed here, there was no difference in the incidence of relapse between patients transplanted in CR and non-CR. This suggests that the patients transplanted in CR were, despite their status, still at a very high relapse risk. Taking these factors into consideration RFS and OS in the current study were acceptable and comparable to the observed outcomes in the Treo/Flu studies examining higher risk patient populations (sAML or active disease at SCT) [11, 32].

The FLAMSA-RIC protocol was designed to reduce AML disease burden prior to transplantation and is often used in patients with active disease. In a retrospective analysis of 60 AML patients (CR 57\%, active disease 43\%) conditioned with FLAMSA-RIC Krejci et al. [33], also observed RFS and OS outcomes comparable to the ones presented here.

The critical period for NRM are the first two years after SCT, in line with the findings of long-term followup studies of post-transplant AML patients $[34,35]$. The NRM after conditioning with Treo/Flu/AraC was $20.1 \%$ at two years. Of note, the addition of cytarabine did not worsen NRM and compared well with previous studies $[9,30]$ in our heavily pre-treated patient population. Besides cytogenetic risk stratification, response to prior treatment reflects the biological behaviour of the patient's disease. As decidedly more patients in this study were not in CR at SCT, NRM rates are higher compared to the studies conducted by Gyurkocza et al. [36] and Deeg et al. [37].

However, our results are comparable to a recent study from the EBMT demonstrating lower 2-year NRM rates for patients with active AML treated with FLAMSA-RIC (7\% vs. 16\% in Bu/Cy, 19\% in Cy/TBI and 18\% in FLAMSA-TBI) and higher 2-year OS (50\% vs. 33\% in Bu/Cy, 34\% in Cy/TBI and 36\% FLMASA-TBI). The two-year cumulative incidence of relapse was $51 \%$ for $\mathrm{Cy} / \mathrm{TBI}, 56 \%$ for Bu/Cy, $55 \%$ for FLAMSA-TBI and $53 \%$ for FLAMSA-RIC [38]. The two-year cumulative incidence of relapse (40.2\%) reported here was acceptable for the high-risk patient population. Two-year relapse rates in the Treo/Flu studies ranged from $16 \%$, in an analysis of 45 patients with primary MDS [30], to $34 \%$ in a study of 75 patients with AML transplanted in CR [19]. Two-year cumulative incidence of relapse in patients transplanted with FLAMSARIC ranged from $22.8 \%$ for high risk AML patients transplanted in CR [39] to $52 \%$ for patients with exclusively primary refractory or relapsed AML [40].

The presence and severity of aGvHD and/or CGvHD is also associated with a reduced risk of AML, CML and MDS relapse [41, 42]. The incidence of II-IV aGvHD is roughly around $35 \%-50 \%$ in all recipients of allogeneic HSCT [43]. The cumulative incidence of grade II-IV aGvHD in the present study (22\%) is low relative to this figure but was comparable to that seen in Treo/Flu and FLAMSA-RIC studies [44, 45]. 
Severe aGvHD was only recorded in $6 \%$ of patients. In addition to the low rate of aGvHD, the cumulative incidence of cGvHD after Treo/Flu/AraC based conditioning was low and potentially contributed to the observed relapse rate.

The cumulative incidence of mild to severe cGvHD at 2 years in the current study was $15 \%$. This figure is low compared to the results of the numerous Treo/Flu and FLAMSA-RIC studies, where the incidence ranged from $24 \%$ [46] to $72 \%$ [8]. The finding might be explained by the immunosuppressive characteristics of treosulfan, which has been shown to result in less proinflammatory cytokine release than busulfan or cyclophosphamide in a mouse model [47]. However, evidence against this theory has been published by Beelen et al., who revealed similar rates for aGvHD and cGvHD, when comparing allogeneic SCT with either Treo/Flu or Bu/Flu conditioning [15]. Attributing the low rates of GvHD found in our study solely to the immunosuppressive effects of treosulfan seems unlikely. Comparing conditioning with cyclophosphamide and TBI with or without cytarabine also found no differences in the incidence of GvHD,effectively excluding the influence of cytarabine to explain the discrepancy [48].

The engraftment and chimerism results show that the Treo/Flu/AraC regimen performs well against other regimens in preparing the host $\mathrm{BM}$ for the engraftment and proliferation of donor stem cells. There was no case of primary or secondary graft failure.

Three cases of SOS (4\%) were reported here. Busulfan-based conditioning regimens are associated with an increased risk of developing SOS [49]. Early phase II Treo/Flu studies did not report any cases of SOS $[19,50]$, suggesting that the use of treosulfan confers a lower risk of this complication. A later retrospective study found an incidence of $2.2 \%$ and two deaths caused by SOS under Treo/Flu conditioning [32]. Having identified patient- and transplant-related risk factors for patients with SOS, the results of this study suggest that the addition of hepatically metabolised cytarabine to the regimen does not dramatically increase the risk of developing SOS.

A grade $3 / 4$ rise in bilirubin and AST/ALT levels exceeded $10 \%$, but on the whole organ toxicities were mild and reversible. Grade 3/4 infections, both sepsis and lung infections exceeded an incidence of $30 \%$. This was as a consequence of the duration and severity of marrow suppression; however, the incidences did not exceed those previously observed in other Treo/Flu or FLAMSA-RIC studies [30, 33, 51].

Nevertheless, the findings of this study have to be interpreted with caution and the retrospective character and the highly heterogeneous patient population should be kept in mind.

In summary, Treo/Flu/AraC is a feasible, effective and safe regimen, which can be used to condition patients unsuitable for myeloablative protocols at high risk of disease relapse prior to allogeneic SCT. The incidence of acute and chronic GVHD is low compared to that seen with the FLAMSA-RIC protocol. Non-haematological toxicities were mild and reversible and RFS and OS were comparable to similar patient groups conditioned with Treo/Flu or FLAMSA-RIC. Compared to the FLAMSA-RIC protocol, Treo/Flu/AraC has a shorter conditioning period with the potential for a reduced duration of hospital stay and shorter neutropenic phase. Large, prospective and randomised controlled trials are required to verify these findings and identify patients who would benefit most from this regimen. The idea of disease- 
specific conditioning protocols, such as cytarabine for AML and melphalan for myeloma, using treosulfan as a backbone should be investigated in the era of personalized medicine.

\section{Declarations}

\section{Acknowledgements}

None

\section{Funding}

This work was made possible by institutional funding.

\section{Competing Interests}

$\mathrm{SH}$ declares no competing interests. IH received speaker fees from Novartis and Jazz and travel support from Medac, Novartis, and Celgene. JF declares no competing interests. AH declares research support from Novartis, BMS, Pfizer and Incyte. C-HK declares honoraria, travel, accommodation and expenses from Bristol-Myers Squibb, Merck, Pfizer, Sanofi and Servier. JC declares honoraria, travel, accommodation and expenses from Medac and Pfizer.

\section{Availability of data and material}

The datasets generated during and/or analysed during the current study are available from the corresponding author on reasonable request.

\section{References}

1. Scott BL, Pasquini MC, Logan BR, Wu J, Devine SM, Porter DL, et al. Myeloablative Versus ReducedIntensity Hematopoietic Cell Transplantation for Acute Myeloid Leukemia and Myelodysplastic Syndromes. J Clin Oncol. 2017;35(11):1154-61. doi: 10.1200/JC0.2016.70.7091.

2. Casper J, Knauf W, Kiefer T, Wolff D, Steiner B, Hammer U, et al. Treosulfan and fludarabine: a new toxicity-reduced conditioning regimen for allogeneic hematopoietic stem cell transplantation. Blood. 2004;103(2):725-31. doi: 10.1182/blood-2002-11-3615.

3. Scheulen ME, Hilger RA, Oberhoff C, Casper J, Freund M, Josten KM, et al. Clinical phase I dose escalation and pharmacokinetic study of high-dose chemotherapy with treosulfan and autologous peripheral blood stem cell transplantation in patients with advanced malignancies. Clin Cancer Res. 2000;6(11):4209-16.

4. Beelen DW, Trenschel R, Casper J, Freund M, Hilger RA, Scheulen ME, et al. Dose-escalated treosulphan in combination with cyclophosphamide as a new preparative regimen for allogeneic haematopoietic stem cell transplantation in patients with an increased risk for regimen-related complications. Bone Marrow Transplant. 2005;35(3):233-41. doi: 10.1038/sj.bmt.1704784. 
5. Munkelt D, Koehl U, Kloess S, Zimmermann SY, Kalaaoui RE, Wehner S, et al. Cytotoxic effects of treosulfan and busulfan against leukemic cells of pediatric patients. Cancer Chemother Pharmacol. 2008;62(5):821-30. doi: 10.1007/s00280-007-0669-3.

6. Gandhi V, Plunkett W. Cellular and clinical pharmacology of fludarabine. Clin Pharmacokinet. 2002;41(2):93-103. doi: 10.2165/00003088-200241020-00002.

7. Ben-Barouch S, Cohen O, Vidal L, Avivi I, Ram R. Busulfan fludarabine vs busulfan cyclophosphamide as a preparative regimen before allogeneic hematopoietic cell transplantation: systematic review and meta-analysis. Bone Marrow Transplant. 2016;51(2):232-40. doi: 10.1038/bmt.2015.238.

8. Hilgendorf I, Wolff D, Gromke T, Trenschel R, Elmaagacli AH, Pichlmeier U, et al. Retrospective analysis of treosulfan-based conditioning in comparison with standard conditioning in patients with myelodysplastic syndrome. Bone Marrow Transplant. 2011;46(4):502-9. doi: 10.1038/bmt.2010.153.

9. Casper J, Wolff D, Knauf W, Blau IW, Ruutu T, Volin L, et al. Allogeneic hematopoietic stem-cell transplantation in patients with hematologic malignancies after dose-escalated treosulfan/fludarabine conditioning. J Clin Oncol. 2010;28(20):3344-51. doi: 10.1200/JCO.2009.23.3429.

10. Schmidt-Hieber M, Blau IW, Trenschel R, Andreesen R, Stuhler G, Einsele H, et al. Reduced-toxicity conditioning with fludarabine and treosulfan prior to allogeneic stem cell transplantation in multiple myeloma. Bone Marrow Transplant. 2007;39(7):389-96. doi: 10.1038/sj.bmt.1705605.

11. Kroger N, Shimoni A, Zabelina T, Schieder H, Panse J, Ayuk F, et al. Reduced-toxicity conditioning with treosulfan, fludarabine and ATG as preparative regimen for allogeneic stem cell transplantation (alloSCT) in elderly patients with secondary acute myeloid leukemia (SAML) or myelodysplastic syndrome (MDS). Bone Marrow Transplant. 2006;37(4):339-44. doi: 10.1038/sj.bmt.1705259.

12. Shimoni A, Rand A, Shem-Tov N, Hardan I, Volchek Y, Yerushalmi R, et al. Fludarabine and Treosulfan Conditioning for Allogeneic Stem-Cell Transplantation; a Dose-Intense Regimen with Limited Toxicity. Blood. 2010;116(21):1427-. doi: 10.1182/blood.V116.21.3473.3473.

13. Shimoni A, Labopin M, Savani B, Hamladji RM, Beelen D, Mufti G, et al. Intravenous Busulfan Compared with Treosulfan-Based Conditioning for Allogeneic Stem Cell Transplantation in Acute Myeloid Leukemia: A Study on Behalf of the Acute Leukemia Working Party of European Society for Blood and Marrow Transplantation. Biol Blood Marrow Transplant. 2018;24(4):751-7. doi: 10.1016/j.bbmt.2017.12.776.

14. Sheth V, Labopin M, Canaani J, Volin L, Brecht A, Ganser A, et al. Comparison of FLAMSA-based reduced intensity conditioning with treosulfan/fludarabine conditioning for patients with acute myeloid leukemia: an ALWP/EBMT analysis. Bone Marrow Transplant. 2019;54(4):531-9. doi: 10.1038/s41409-018-0288-0.

15. Beelen DW, Trenschel R, Stelljes M, Groth C, Masszi T, Remenyi P, et al. Treosulfan or busulfan plus fludarabine as conditioning treatment before allogeneic haemopoietic stem cell transplantation for older patients with acute myeloid leukaemia or myelodysplastic syndrome (MC-FludT.14/L): a 
randomised, non-inferiority, phase 3 trial. Lancet Haematol. 2020;7(1):e28-e39. doi: 10.1016/S23523026(19)30157-7.

16. Capizzi RL. Curative chemotherapy for acute myeloid leukemia: the development of high-dose ara-C from the laboratory to bedside. Invest New Drugs. 1996;14(3):249-56. doi: 10.1007/BF00194527.

17. McLaughlin B, Im A, Raptis A, Agha M, Hou JZ, Redner R, et al. Fludarabine and cytarabine in patients with relapsed acute myeloid leukemia refractory to initial salvage therapy. Int $\mathrm{J}$ Hematol. 2012;96(6):743-7. doi: 10.1007/s12185-012-1192-9.

18. Gandhi V, Estey E, Keating MJ, Plunkett W. Fludarabine potentiates metabolism of cytarabine in patients with acute myelogenous leukemia during therapy. J Clin Oncol. 1993;11(1):116-24. doi: 10.1200/JC0.1993.11.1.116.

19. Casper J, Holowiecki J, Trenschel R, Wandt H, Schaefer-Eckart K, Ruutu T, et al. Allogeneic hematopoietic SCT in patients with AML following treosulfan/fludarabine conditioning. Bone Marrow Transplant. 2012;47(9):1171-7. doi: 10.1038/bmt.2011.242.

20. Ruutu T, Juvonen E, Remberger M, Remes K, Volin L, Mattsson J, et al. Improved survival with ursodeoxycholic acid prophylaxis in allogeneic stem cell transplantation: long-term follow-up of a randomized study. Biol Blood Marrow Transplant. 2014;20(1):135-8. doi: 10.1016/j.bbmt.2013.10.014.

21. Olsson R, Remberger M, Schaffer M, Berggren DM, Svahn BM, Mattsson J, et al. Graft failure in the modern era of allogeneic hematopoietic SCT. Bone Marrow Transplant. 2013;48(4):537-43. doi: 10.1038/bmt.2012.239.

22. Larocca A, Piaggio G, Podesta M, Pitto A, Bruno B, Di Grazia C, et al. Boost of CD34+-selected peripheral blood cells without further conditioning in patients with poor graft function following allogeneic stem cell transplantation. Haematologica. 2006;91(7):935-40.

23. Olsson RF, Logan BR, Chaudhury S, Zhu X, Akpek G, Bolwell BJ, et al. Primary graft failure after myeloablative allogeneic hematopoietic cell transplantation for hematologic malignancies. Leukemia. 2015;29(8):1754-62. doi: 10.1038/leu.2015.75.

24. Harris AC, Young R, Devine S, Hogan WJ, Ayuk F, Bunworasate U, et al. International, Multicenter Standardization of Acute Graft-versus-Host Disease Clinical Data Collection: A Report from the Mount Sinai Acute GVHD International Consortium. Biol Blood Marrow Transplant. 2016;22(1):4-10. doi: 10.1016/j.bbmt.2015.09.001.

25. Filipovich AH, Weisdorf D, Pavletic S, Socie G, Wingard JR, Lee SJ, et al. National Institutes of Health consensus development project on criteria for clinical trials in chronic graft-versus-host disease: I. Diagnosis and staging working group report. Biol Blood Marrow Transplant. 2005;11(12):945-56. doi: 10.1016/j.bbmt.2005.09.004.

26. Schemper M, Smith TL. A note on quantifying follow-up in studies of failure time. Control Clin Trials. 1996;17(4):343-6. doi: 10.1016/0197-2456(96)00075-x.

27. Scrucca L, Santucci A, Aversa F. Competing risk analysis using R: an easy guide for clinicians. Bone Marrow Transplant. 2007;40(4):381-7. doi: 10.1038/sj.bmt.1705727. 
28. Sorror ML, Maris MB, Storb R, Baron F, Sandmaier BM, Maloney DG, et al. Hematopoietic cell transplantation (HCT)-specific comorbidity index: a new tool for risk assessment before allogeneic HCT. Blood. 2005;106(8):2912-9. doi: 10.1182/blood-2005-05-2004.

29. Corbacioglu S, Jabbour EJ, Mohty M. Risk Factors for Development of and Progression of Hepatic Veno-Occlusive Disease/Sinusoidal Obstruction Syndrome. Biol Blood Marrow Transplant. 2019;25(7):1271-80. doi: 10.1016/j.bbmt.2019.02.018.

30. Ruutu T, Volin L, Beelen DW, Trenschel R, Finke J, Schnitzler M, et al. Reduced-toxicity conditioning with treosulfan and fludarabine in allogeneic hematopoietic stem cell transplantation for myelodysplastic syndromes: final results of an international prospective phase II trial. Haematologica. 2011;96(9):1344-50. doi: 10.3324/haematol.2011.043810.

31. Michallet M, Sobh M, Milpied N, Bay JO, Furst S, Harousseau JL, et al. Phase II prospective study of treosulfan-based reduced-intensity conditioning in allogeneic HSCT for hematological malignancies from 10/10 HLA-identical unrelated donor. Ann Hematol. 2012;91(8):1289-97. doi: 10.1007/s00277012-1429-y.

32. Nagler A, Labopin M, Beelen D, Ciceri F, Volin L, Shimoni A, et al. Long-term outcome after a treosulfan-based conditioning regimen for patients with acute myeloid leukemia: A report from the Acute Leukemia Working Party of the European Society for Blood and Marrow Transplantation. Cancer. 2017;123(14):2671-9. doi: 10.1002/cncr.30646.

33. Krejci M, Doubek M, Dusek J, Brychtova Y, Racil Z, Navratil M, et al. Combination of fludarabine, amsacrine, and cytarabine followed by reduced-intensity conditioning and allogeneic hematopoietic stem cell transplantation in patients with high-risk acute myeloid leukemia. Ann Hematol. 2013;92(10):1397-403. doi: 10.1007/s00277-013-1790-5.

34. Shimoni A, Labopin M, Savani B, Volin L, Ehninger G, Kuball J, et al. Long-term survival and late events after allogeneic stem cell transplantation from HLA-matched siblings for acute myeloid leukemia with myeloablative compared to reduced-intensity conditioning: a report on behalf of the acute leukemia working party of European group for blood and marrow transplantation. J Hematol Oncol. 2016;9(1):118. doi: 10.1186/s13045-016-0347-1.

35. Socie G, Stone JV, Wingard JR, Weisdorf D, Henslee-Downey PJ, Bredeson C, et al. Long-term survival and late deaths after allogeneic bone marrow transplantation. Late Effects Working Committee of the International Bone Marrow Transplant Registry. N Engl J Med. 1999;341(1):14-21. doi: 10.1056/NEJM199907013410103.

36. Gyurkocza B, Gutman J, Nemecek ER, Bar M, Milano F, Ramakrishnan A, et al. Treosulfan, fludarabine, and 2-Gy total body irradiation followed by allogeneic hematopoietic cell transplantation in patients with myelodysplastic syndrome and acute myeloid leukemia. Biol Blood Marrow Transplant. 2014;20(4):549-55. doi: 10.1016/j.bbmt.2014.01.009.

37. Deeg HJ, Stevens EA, Salit RB, Ermoian RP, Fang M, Gyurkocza B, et al. Transplant Conditioning with Treosulfan/Fludarabine with or without Total Body Irradiation: A Randomized Phase II Trial in 
Patients with Myelodysplastic Syndrome and Acute Myeloid Leukemia. Biol Blood Marrow Transplant. 2018;24(5):956-63. doi: 10.1016/j.bbmt.2017.12.785.

38. Rodriguez-Arboli E, Labopin M, Tischer J, Brecht A, Ganser A, Finke J, et al. FLAMSA-Based ReducedIntensity Conditioning versus Myeloablative Conditioning in Younger Patients with Relapsed/Refractory Acute Myeloid Leukemia with Active Disease at the Time of Allogeneic Stem Cell Transplantation: An Analysis from the Acute Leukemia Working Party of the European Society for Blood and Marrow Transplantation. Biol Blood Marrow Transplant. 2020;26(11):2165-73. doi: 10.1016/j.bbmt.2020.07.020.

39. Malard F, Labopin M, Stuhler G, Bittenbring J, Ganser A, Tischer J, et al. Sequential Intensified Conditioning Regimen Allogeneic Hematopoietic Stem Cell Transplantation in Adult Patients with Intermediate- or High-Risk Acute Myeloid Leukemia in Complete Remission: A Study from the Acute Leukemia Working Party of the European Group for Blood and Marrow Transplantation. Biol Blood Marrow Transplant. 2017;23(2):278-84. doi: 10.1016/j.bbmt.2016.11.002.

40. Schneidawind D, Federmann B, Faul C, Vogel W, Kanz L, Bethge WA. Allogeneic hematopoietic cell transplantation with reduced-intensity conditioning following FLAMSA for primary refractory or relapsed acute myeloid leukemia. Ann Hematol. 2013;92(10):1389-95. doi: 10.1007/s00277-0131774-5.

41. Patel H, Molina A, Nikanjam M, Schiller GJ. Risk Factors for Relapse Following Allogeneic Transplant for Acute Myeloid Leukemia in the UCLA Patient Population. Blood. 2016;128(22). doi: DOI 10.1182/blood.V128.22.5855.5855.

42. Stern M, de Wreede LC, Brand R, van Biezen A, Dreger P, Mohty M, et al. Impact of Graft-Versus-Host Disease On Relapse After Allogeneic Hematopoietic Stem Cell Transplantation, an EBMT Megafile Study. Blood. 2012;120(21):469-. doi: 10.1182/blood.V120.21.469.469.

43. Jacobsohn DA, Vogelsang GB. Acute graft versus host disease. Orphanet J Rare Dis. 2007;2(1):35. doi: 10.1186/1750-1172-2-35.

44. Remberger M, Torlen J, Serafi IE, Garming-Legert K, Bjorklund A, Ljungman P, et al. Toxicological effects of fludarabine and treosulfan conditioning before allogeneic stem-cell transplantation. Int $\mathrm{J}$ Hematol. 2017;106(4):471-5. doi: 10.1007/s12185-017-2320-3.

45. Pfrepper C, Klink A, Behre G, Schenk T, Franke GN, Jentzsch M, et al. Risk factors for outcome in refractory acute myeloid leukemia patients treated with a combination of fludarabine, cytarabine, and amsacrine followed by a reduced-intensity conditioning and allogeneic stem cell transplantation. J Cancer Res Clin Oncol. 2016;142(1):317-24. doi: 10.1007/s00432-015-2050-y.

46. Baronciani D, Rambaldi A, lori AP, Di Bartolomeo P, Pilo F, Pettinau M, et al. Treosulfan/fludarabine as an allogeneic hematopoietic stem cell transplant conditioning regimen for high-risk patients. Am J Hematol. 2008;83(9):717-20. doi: 10.1002/ajh.21240.

47. Sjoo F, Hassan Z, Abedi-Valugerdi M, Griskevicius L, Nilsson C, Remberger M, et al. Myeloablative and immunosuppressive properties of treosulfan in mice. Exp Hematol. 2006;34(1):115-21. doi: 10.1016/j.exphem.2005.09.015. 
48. Arai Y, Aoki K, Takeda J, Kondo T, Eto T, Ota S, et al. Clinical significance of high-dose cytarabine added to cyclophosphamide/total-body irradiation in bone marrow or peripheral blood stem cell transplantation for myeloid malignancy. J Hematol Oncol. 2015;8(1):102. doi: 10.1186/s13045-0150201-x.

49. Dix SP, Wingard JR, Mullins RE, Jerkunica I, Davidson TG, Gilmore CE, et al. Association of busulfan area under the curve with veno-occlusive disease following BMT. Bone Marrow Transplant. 1996;17(2):225-30.

50. Casper J, Knauf W, Blau I, Ruutu T, Volin L, Wandt H, et al. Treosulfan/fludarabine: a new conditioning regimen in allogeneic transplantation. Ann Hematol. 2004;83 Suppl 1:S70-1. doi: 10.1007/s00277-004-0850-2.

51. Casper J, Freund M. Treosulfan based conditioning for autologous and allogeneic hematopoietic stem cell transplantation. Int J Clin Pharmacol Ther. 2004;42(11):661-2. doi: 10.5414/cpp42661.

\section{Tables}


Table 1

Characteristics of all 77 patients and donors

\begin{tabular}{|c|c|}
\hline Patient or Graft (Donor) Characteristic & Number (Percentage) \\
\hline Patient age at transplant median (Range), years & 54 years (18-69 years) \\
\hline \multicolumn{2}{|l|}{ Patient's sex } \\
\hline Male & $52(68 \%)$ \\
\hline Female & $25(32 \%)$ \\
\hline Female donor for male recipient & $17(21 \%)$ \\
\hline \multicolumn{2}{|l|}{ Diagnosis at time of transplantation } \\
\hline Primary acute myeloid leukaemia & $33(43 \%)$ \\
\hline Secondary acute myeloid leukaemia & $25(32 \%)$ \\
\hline Myelodysplastic syndrome & $6(8 \%)$ \\
\hline Myeloproliferative neoplasm & $13(17 \%)$ \\
\hline \multicolumn{2}{|l|}{ Primary AML ELN risk stratification by genetics (53) } \\
\hline Favourable & $5(15 \%)$ \\
\hline Intermediate & $20(61 \%)$ \\
\hline Adverse & $8(24 \%)$ \\
\hline \multicolumn{2}{|l|}{ Status at transplantation } \\
\hline CR1 & $20(25 \%)$ \\
\hline CR2 & $13(16 \%)$ \\
\hline$\geq \mathrm{CR} 3$ & $3(4 \%)$ \\
\hline First or second partial response & $12(15 \%)$ \\
\hline Relapse & $6(7.5 \%)$ \\
\hline Progressive disease & $14(18 \%)$ \\
\hline First or second chronic phase & $5(6 \%)$ \\
\hline First blast crisis & $1(1 \%)$ \\
\hline Stable disease & $6(7.5 \%)$ \\
\hline \multicolumn{2}{|l|}{ CR1 (breakdown of diagnoses within this group) } \\
\hline Secondary AML & 9 \\
\hline Primary AML & 8 \\
\hline
\end{tabular}




\begin{tabular}{|c|c|}
\hline - Adverse genetic risk (ELN) & 4 \\
\hline - Intermediate genetic risk (ELN) * & 4 \\
\hline High-risk CML (ELTS score) & 3 \\
\hline \multicolumn{2}{|c|}{ Treatment before transplantation (no. of courses) } \\
\hline None & $2(3 \%)$ \\
\hline One & $25(31 \%)$ \\
\hline Two & $32(40 \%)$ \\
\hline$>$ Two & $21(26 \%)$ \\
\hline Previous allogeneic SCT & $10(13 \%)$ \\
\hline \multicolumn{2}{|l|}{ CMV status (patient/donor) } \\
\hline Negative/negative & $26(32.5 \%)$ \\
\hline Negative/positive & $9(11 \%)$ \\
\hline Positive/negative & $18(22.5 \%)$ \\
\hline Positive/positive & $27(34 \%)$ \\
\hline \multicolumn{2}{|l|}{ Donor } \\
\hline Matched related donor (MRD) & 21 (26\%) \\
\hline Matched unrelated donor (MUD) & $49(61 \%)$ \\
\hline Mismatched unrelated donor (MMUD) & $10(13 \%)$ \\
\hline \multicolumn{2}{|l|}{ Stem cell source } \\
\hline Bone marrow & $2(2.5 \%)$ \\
\hline Mobilised peripheral blood stem cells & $78(97.5 \%)$ \\
\hline \multicolumn{2}{|c|}{ Hematopoietic cell transplantation-specific comorbidity index (HCT-Cl) } \\
\hline \multicolumn{2}{|l|}{0} \\
\hline $1-2$ & $48(60 \%)$ \\
\hline \multirow[t]{2}{*}{$>2$} & $20(25 \%)$ \\
\hline & 12 (15\%) \\
\hline
\end{tabular}

$\mathrm{AML}$, acute myeloid leukemia; CMV, cytomegalovirus; CR, complete remission; CR1/2/3, first/second/third complete remission; ELN, European LeukemiaNet; ELTS, EUTOS long-term survival; HCT-Cl, Hematopoietic Cell Transplantation-specific Comorbidity Index; MMUD, mismatched unrelated donor; MRD, matchedrelated donor; MUD, matched-unrelated donor; SCT, stem cell transplantation. 
Table 2

Overview of the NRM rates for all patients and group analyses

\begin{tabular}{|lllll|}
\hline $\begin{array}{l}\text { Patient Groups (number of transplanted } \\
\text { patients) }\end{array}$ & \multicolumn{3}{l}{ Non-Relapse Mortality (\%) } & $\begin{array}{c}\text { P-value } \\
\text { (Gray's test) }\end{array}$ \\
& $\frac{100}{\text { day } \underline{\underline{s}}}$ & $\underline{\text { One-year }}$ & $\underline{\text { Two-year }}$ & - \\
\hline All patients (77) & 10.0 & 18.8 & 20.1 & \\
\hline MUD (48) & 12.0 & 16.0 & 16.0 & 0.31 \\
MRD (20) & 0.0 & 19.0 & 24.0 & \\
MMUD (9) & 20.0 & 20.0 & 30.0 & \\
\hline CR (35) & 5.4 & 16.2 & 18.9 & 0.91 \\
Non-CR (42) & 14.0 & 20.9 & 20.9 & \\
\hline$<50$ years (25) & 0.0 & 11.0 & 11.0 & 0.077 \\
$\geq 50$ years (52) & 15.0 & 23.0 & 25.0 & \\
\hline AML patients only (58) & 11.7 & 21.7 & 21.7 & \\
\hline
\end{tabular}

$A M L$, acute myeloid leukemia; $C R$, complete remission; MMUD, mismatched unrelated donor; MRD, matched-related donor; MUD, matched-unrelated donor. 
Table 3

Overview of the relapse rates for all patients and group analyses

\begin{tabular}{|c|c|c|c|}
\hline \multirow[t]{2}{*}{ Patient Groups (number of transplanted patients) } & \multicolumn{2}{|c|}{ Rate of Relapse (\%) } & \multirow{2}{*}{$\begin{array}{l}\text { P-value } \\
\text { (Gray's test) } \\
\text { - }\end{array}$} \\
\hline & One-year & Three-year & \\
\hline All patients (80) & 34.0 & 41.0 & \\
\hline MUD (49) & 37.0 & 48.0 & 0.25 \\
\hline MRD (21) & 29.0 & 29.0 & \\
\hline MMUD (10) & 30.0 & 30.0 & \\
\hline CR (37) & 35.1 & 43.6 & 0.92 \\
\hline Non-CR (43) & 32.6 & 38.7 & \\
\hline$<50$ years $(27)$ & 44.0 & 55.0 & 0.08 \\
\hline$\geq 50$ years $(53)$ & 28.0 & 32.0 & \\
\hline AML patients only (58) & 35.0 & 42.5 & \\
\hline
\end{tabular}

$A M L$, acute myeloid leukemia; $C R$, complete remission; MMUD, mismatched unrelated donor; MRD, matched-related donor; MUD, matched-unrelated donor. 
Table 4

Toxicities and adverse events that occurred during conditioning up until day +28 following transplantation, graded according to the Common Terminology Criteria for Adverse Events (CTCAE) version 5.0

\begin{tabular}{|c|c|}
\hline Adverse Event & Number of Patients, $n$ (\%) \\
\hline \multicolumn{2}{|l|}{ Oral mucositis } \\
\hline Grade 1-2 & $14(18)$ \\
\hline Grade 3-4 & $18(23)$ \\
\hline \multicolumn{2}{|c|}{ Creatinine increase } \\
\hline Grade 1-2 & $34(44)$ \\
\hline Grade 3-4 & $1(1.3)$ \\
\hline \multicolumn{2}{|c|}{ Sinusoidal obstruction syndrome } \\
\hline Grade 3-4 & $2(2.6)$ \\
\hline Grade 5 & $1(1.3)$ \\
\hline \multicolumn{2}{|c|}{ AST/ALT increase } \\
\hline Grade 1 & $33(43)$ \\
\hline Grade 2 & $22(29)$ \\
\hline Grade 3-4 & $17(22)$ \\
\hline \multicolumn{2}{|l|}{ AP increase } \\
\hline Grade 1-2 & $45(58)$ \\
\hline Grade 3-4 & $3(3.9)$ \\
\hline \multicolumn{2}{|c|}{ Bilirubin increase } \\
\hline Grade 1-2 & $52(68)$ \\
\hline Grade 3-4 & $12(16)$ \\
\hline \multicolumn{2}{|c|}{ Febrile neutropenia } \\
\hline Grade 3-4 & $48(62)$ \\
\hline \multicolumn{2}{|l|}{ Sepsis } \\
\hline Grade 3-4 & $32(42)$ \\
\hline Grade 5 & $2(2.6)$ \\
\hline \multicolumn{2}{|l|}{ Lung infection } \\
\hline Grade 3-4 & $27(34)$ \\
\hline
\end{tabular}


Figures

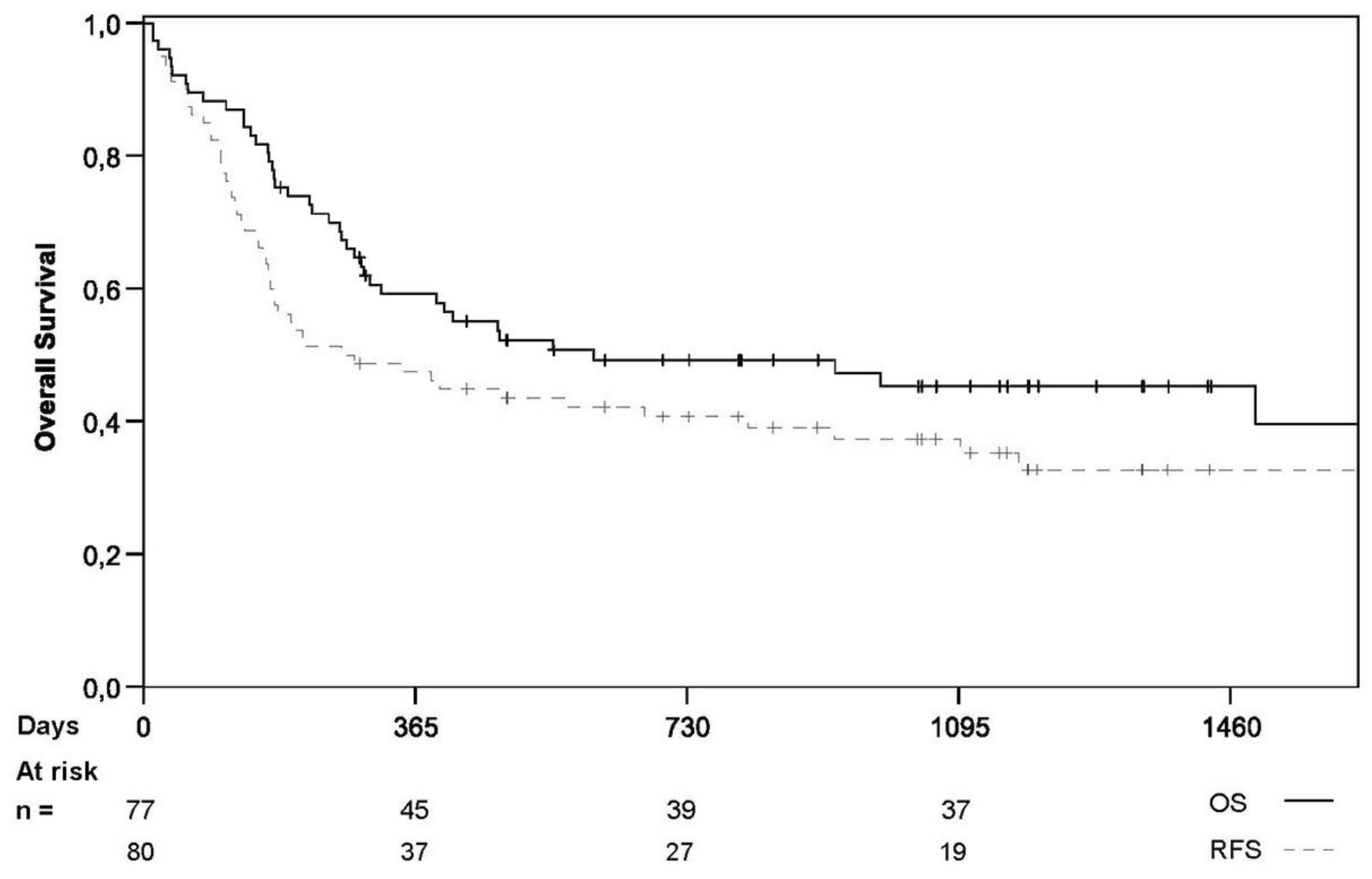

\section{Figure 1}

Overall survival estimates (full line) and relapse-free survival estimates (broken line), calculated using the Kaplan-Meier method, for all 77 patients conditioned with Treo/Flu/AraC. Small vertical lines denote a censored event 


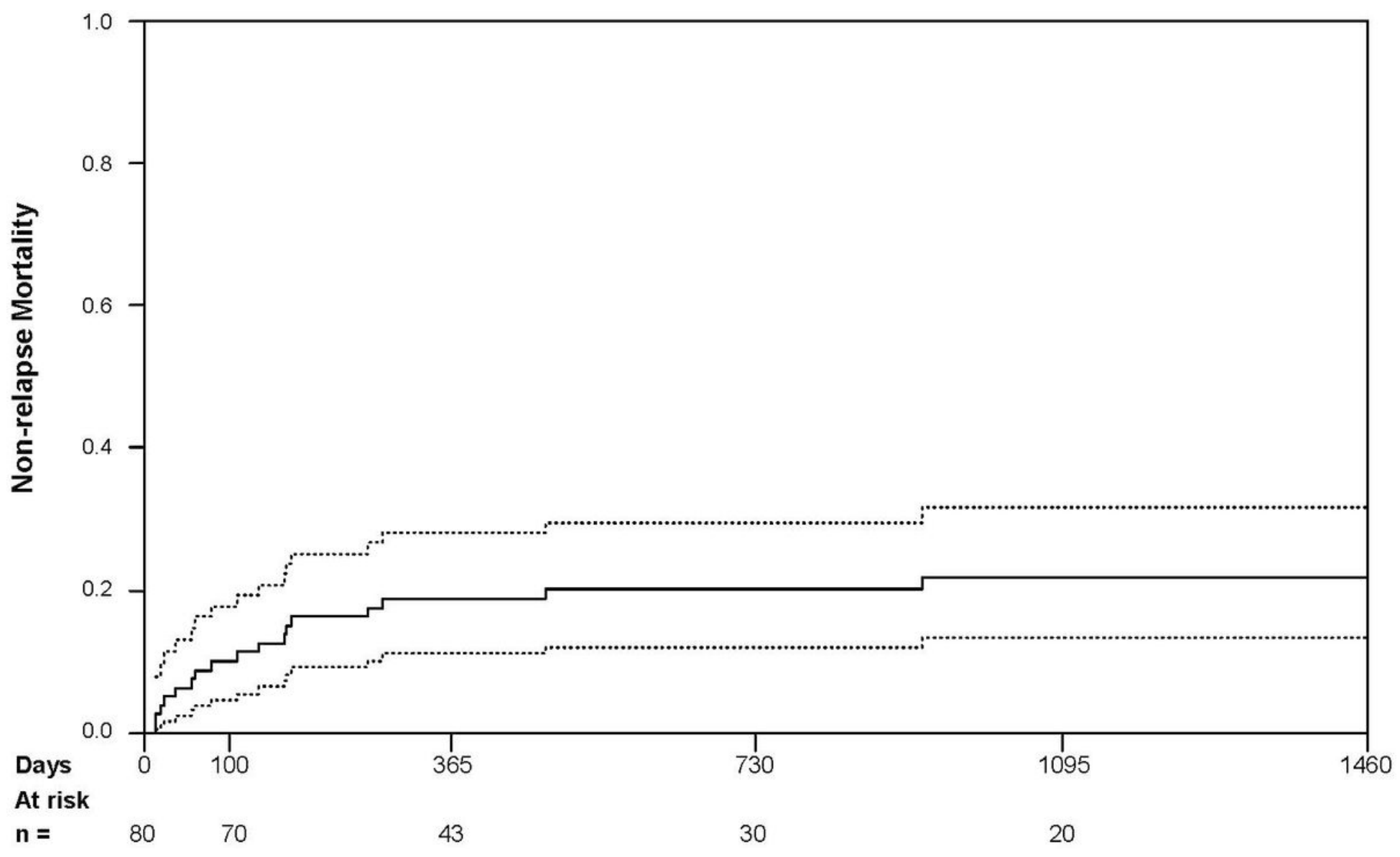

Figure 2

Cumulative incidence of non-relapse mortality for all patients (full line) with $95 \%$ confidence intervals (broken line) 


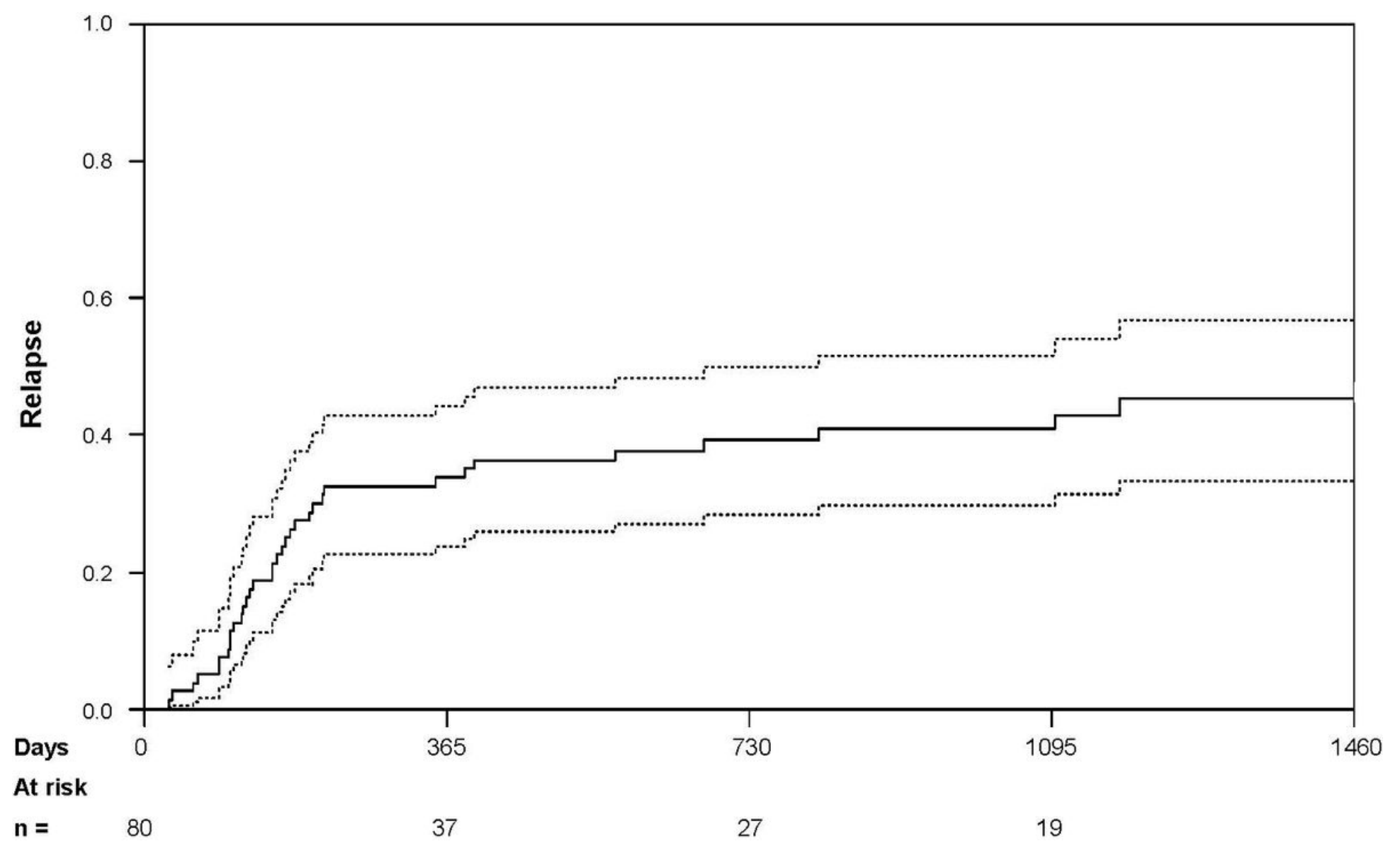

Figure 3

Cumulative incidence of relapse for all patients (full line) with $95 \%$ confidence intervals (broken line)

\section{Supplementary Files}

This is a list of supplementary files associated with this preprint. Click to download.

- SupplementaltablesV4.docx 